\title{
RegentK: The Scientific Investigation of a 40-Year-Old Method in Regenerative Medicine
}

\author{
Gerhard Litscher
}

Research Unit for Complementary and Integrative Laser Medicine, Research Unit of Biomedical Engineering in Anesthesia and Intensive Care Medicine, and TCM Research Center Graz, Medical University of Graz, Graz, Austria

\section{Key Words}

RegentK · Anterior cruciate ligament · Manual therapy · Near-infrared spectroscopy ·

Thermal imaging $\cdot$ Electrodermal mapping

\section{Abstract}

Background: Mohamed Khalifa has been healing even the most severe muscle and joint injuries in Austria for more than 40 years, without surgery, only with his hands by applying pressure on the skin. In this article, the author summarises the first scientific results trying to describe the way of functioning of this unusual form of therapy called RegentK. Historical aspects, regional oxygen saturation of deeper knee tissues, thermographic measurements, and electrodermal mapping are described in detail. Summary: Rupture of the anterior cruciate ligament $(\mathrm{ACL})$ is a high-incidence injury usually treated surgically. According to common knowledge, it does not heal spontaneously, although some claim the opposite. RegentK was developed for injuries of the musculoskeletal system. In one study, it was shown that after 3 months, $47 \%$ of the RegentK patients, but no physiotherapy patient, demonstrated an endto-end homogeneous ACL on magnetic resonance imaging. Key Messages: We conclude that $\mathrm{ACL}$ healing can be improved with RegentK.

(C) 2015 S. Karger AG, Basel

\section{Introduction}

The native Egyptian Mohamed Khalifa has been healing even the most severe muscle and joint injuries (e.g. rupture of the cruciate ligament) in Austria for more than 40 years, without surgery, only with his hands by applying pressure on the skin, in just one hour; completely and permanently (fig. 1) [1]. 
Litscher et al.: RegentK: The Scientific Investigation of a 40 -Year-Old Method in Regenerative Medicine

Fig. 1. RegentK therapy by Mohamed Khalifa (2013).

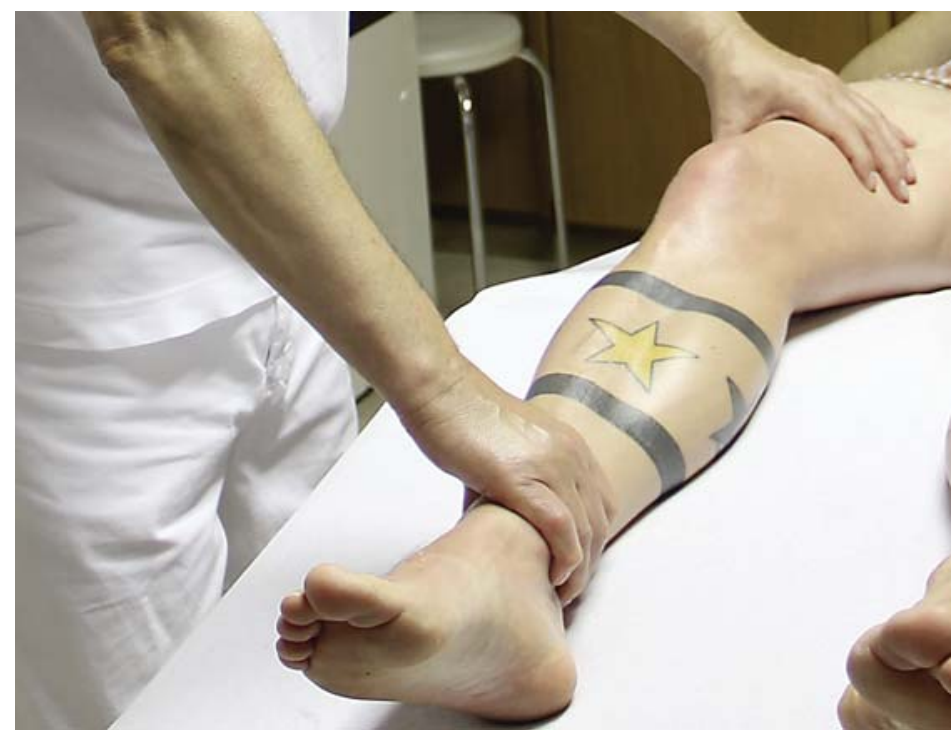

He treats about 3 patients per day and has unusually high success rates. His patients include top athletes, like Steffi Graf, Roger Federer, Boris Becker, Marc Girardelli, and Sergey Bubka, as well as footballers, skiers, climbers, and even trauma surgeons themselves [1].

It seems possible that Mohamed Khalifa has found a new method in medicine. In this review article, the author summarises the first scientific results trying to describe the way of functioning of this unusual form of therapy called RegentK. The goal of this comprehensive article is to provide information to all interested persons in the scientific community.

\section{Historical Aspects}

The knee is a complicated joint with multiple ligaments (fig. 2). The cruciate ligaments secure the stability in the knee in cooperation with other ligaments, the menisci, and muscles. Injuries of the anterior cruciate ligament (ACL) are more common than injuries of the posterior cruciate ligament. Already in 1949, Tidy [2] made the following statement concerning a rupture of the ACL in Massage and Remedial Exercises in Medical and Surgical Conditions:

'The injury is a very serious one, since, unless the crucial ligaments heal well and firmly, the knee will always be unstable. For this reason the joint is invariably immobilized for a long period. Six weeks at least are necessary for the complete repair of ligaments, and in such a joint as the knee, which depends for its stability on its ligaments rather than the conformation of its bony surfaces, it is well to allow a little extra time.'

Tidy [2] further observed:

'On the crucial ligaments the stability of the knee largely depends. If both are injured, the knee hyperextends, and there is abnormal lateral mobility. If the anterior one alone suffers, the tibia can be moved backwards and forwards on the femur, especially in the flexed position. In either case the knee is rendered weak and undependable, and is liable to give way when weight is placed on it.'

Gisbert Niederführ, author of the book Torn Ligaments? A Slipped Disc? Operations No Longer Necessary! [3], knows Khalifa very well. He summarised details of the history and technique of Mohamed Khalifa: 
Fig. 2. Illustration of the ligaments of the right knee, including the anterior and posterior cruciate ligaments.
Litscher et al.: RegentK: The Scientific Investigation of a $40-$ Year-Old Method in Regenerative Medicine

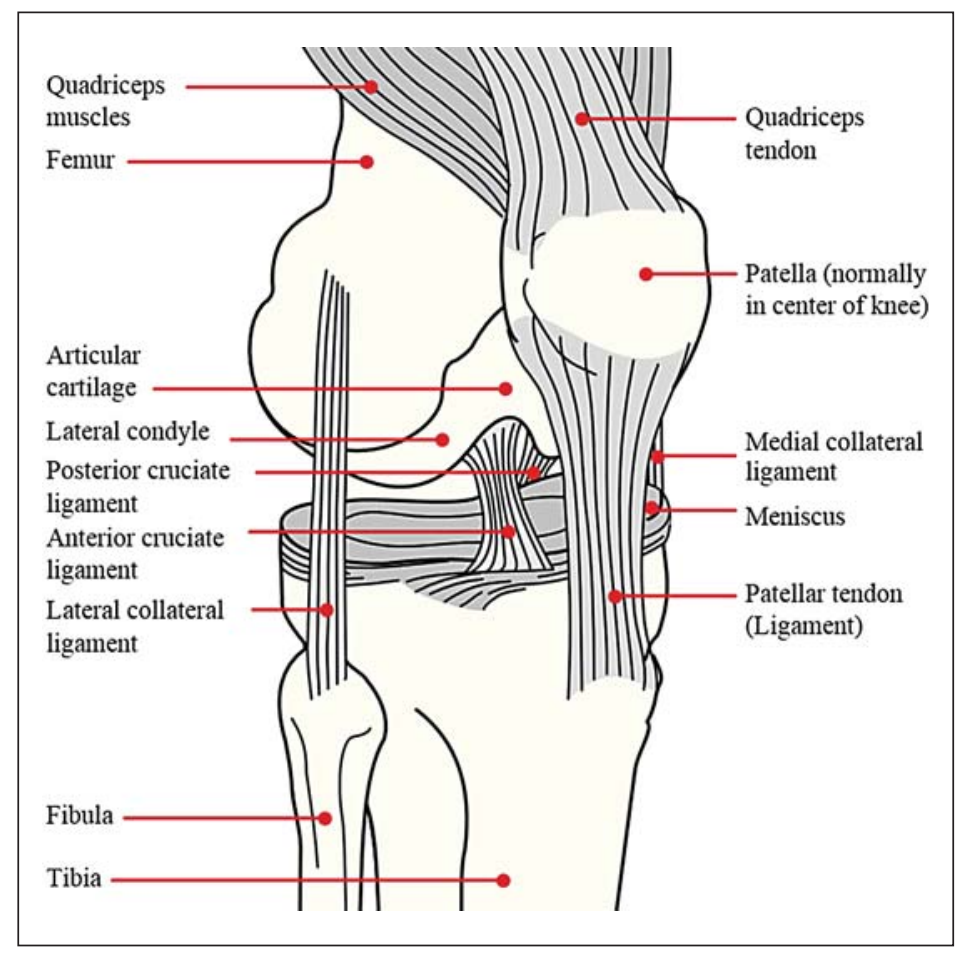

'Mohamed Khalifa's method is based on the new physics and endeavours not to damage the body but to help it and support it in its own, natural healing activities. If you cut through an elastic band and sew it together again, you cannot expect it to be as elastic at the stitching point as it was before. With human ligaments it's the same. And if the elasticity is disrupted at any point in the human body, the whole system is affected. Mohamed Khalifa says: "Elasticity is a part of human energy. The flow of energy through the whole body must not be disrupted." Doctors, orthopaedists and surgeons operate with the best of intentions and treat their patients with perfect precision - perfect from the anatomical-pathological standpoint which they represent. Khalifa describes his method as functional-pathological. In the anatomicalpathological approach of modern medical science, a pathological (ailing) part is simply replaced in order to restore the body anatomically. Not so with Khalifa. For him function is the primary concern, not anatomy. The most important thing is not the crucial ligament itself but the function it carries out. After all, what the patient is primarily interested in is that his body functions properly again. Mohamed Khalifa restores the function of the knee in a natural way. Nothing is destroyed here, but healed - healed fully, without any consequential injuries. "Healing," says Khalifa, "is restoring form and function." Form is growth achieved by nutrients, nutrients supplied by the trophic metabolism. And in the last resort, healing is nothing else but growth - new growth, a growth that Khalifa's hands foster and promote' [3].

\section{Regional Oxygen Saturation of Deeper Knee Tissues}

Up to now, it is still unknown whether the microcirculation of deeper peripheral tissue (3-4 cm) can be modulated by pressure, acupressure, or acupuncture on an acupuncture point. The goal of a pilot study was to investigate possible effects of acupressure at the Xiyangguan acupoint (GB33) on the regional oxygen saturation $\left(\mathrm{rSO}_{2}\right)$ of the deeper knee tissues by near-infrared spectroscopy [4]. Therefore, 12 healthy volunteers with a mean \pm SD age of $23.8 \pm 1.6$ years were investigated. Acupressure stimulation was performed for $5 \mathrm{~min}$ at the acupoint. The results of the controlled study showed a significant increase in the values of $\mathrm{rSO}_{2}$ on the stimulated side of the knee ( $\mathrm{p}=0.033$ ), whereas the opposite side of the same 
Litscher et al.: RegentK: The Scientific Investigation of a 40-Year-Old Method in Regenerative Medicine

Fig. 3. Four near-infrared spectroscopy sensors on the knees in a patient with a rupture of the ACL.

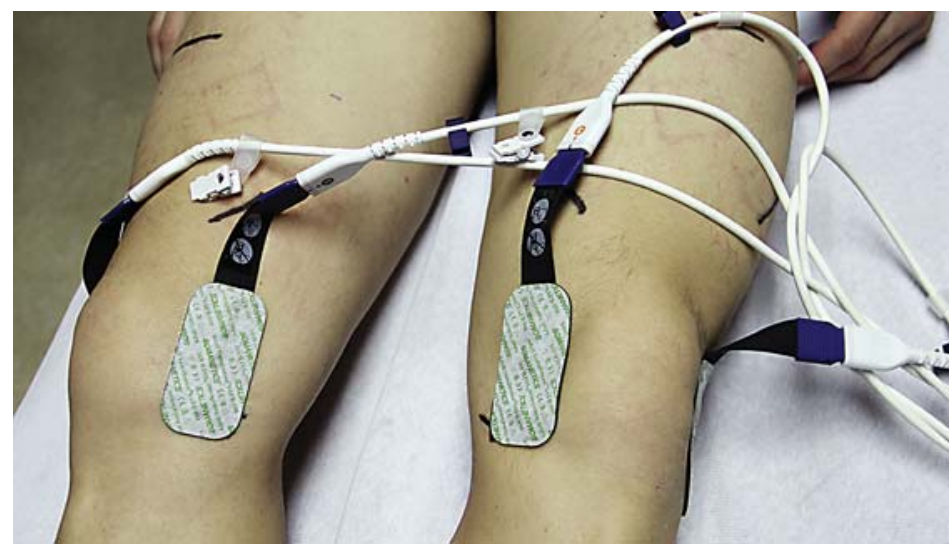

knee showed insignificant changes. These results served as a valuable basis for monitoring a possible therapeutic effect (e.g. of RegentK) in patients with knee injuries [4].

Manual Khalifa therapy or RegentK has been practiced in Hallein, Austria, for several decades; however, previously, there were no scientific results available on the topic. The goal of a recently published study [5] was to investigate possible acute effects of this unique therapy on $\mathrm{rSO}_{2}$ of knee tissues in patients with completely ruptured ACL. We investigated 10 male patients (mean age \pm SD $35.9 \pm 6.1$ years) using a four-channel INVOS 5100C oximeter (Somanetics, Troy, Mich., USA). The sensors were applied anterolaterally and anteromedially, beside the patella, on both the injured and the healthy (control) knee (fig. 3). The results of this controlled study showed that $\mathrm{rSO}_{2}$ values on the knee with the ruptured ligament were significantly increased ( $\mathrm{p}<0.001)$ immediately after RegentK, whereas the values on the control knee showed insignificant increases. Baseline values of the anterolateral side of the injured knee were significantly $(p<0.001)$ different from those of the anterolateral side of the control knee. The same effect was present on the anteromedial side, however, with a lower degree of significance $(\mathrm{p}<0.05)$. RegentK was clinically successful in all 10 patients [5].

The goal of another study from our research team was to again investigate acute effects of RegentK compared to standard physiotherapy on $\mathrm{rSO}_{2}$ of knee tissues in patients with completely ruptured ACL. In this study, 20 patients were enrolled and randomly assigned to receive either RegentK (group A; 10 patients, mean age \pm SD $31.3 \pm 8.5$ years, 8 female, 2 male) or physiotherapy (group B; 10 patients, mean age \pm SD $34.8 \pm 10.2$ years, 6 female, 4 male). $\mathrm{rSO}_{2}$ increased significantly ( $\mathrm{p}<0.001$ ) immediately after RegentK on both registration sites of the injured knee, whereas after physiotherapy, only the anterolateral side showed significant increases (fig. 4). Interestingly, we found significant increases in $\mathrm{rSO}_{2}$ also on the control knee after RegentK; in group B, these results were insignificant. Based on this study, we concluded that manual therapy influences the hemodynamics of muscles and deeper structures. The results also serve to quantify the effects of non-pharmacological and non-surgical interventions on the microvascular circulation in deep tissue after a complete rupture of the ACL [6].

\section{Thermographic Measurements}

One preliminary publication on the topic of RegentK describes acute temperature effects after this therapy. The goal of this study was to describe the temperature distribution and the effects on the surface temperature of the knees and feet in patients with completely ruptured 
Litscher et al.: RegentK: The Scientific Investigation of a 40-Year-Old Method in Regenerative Medicine

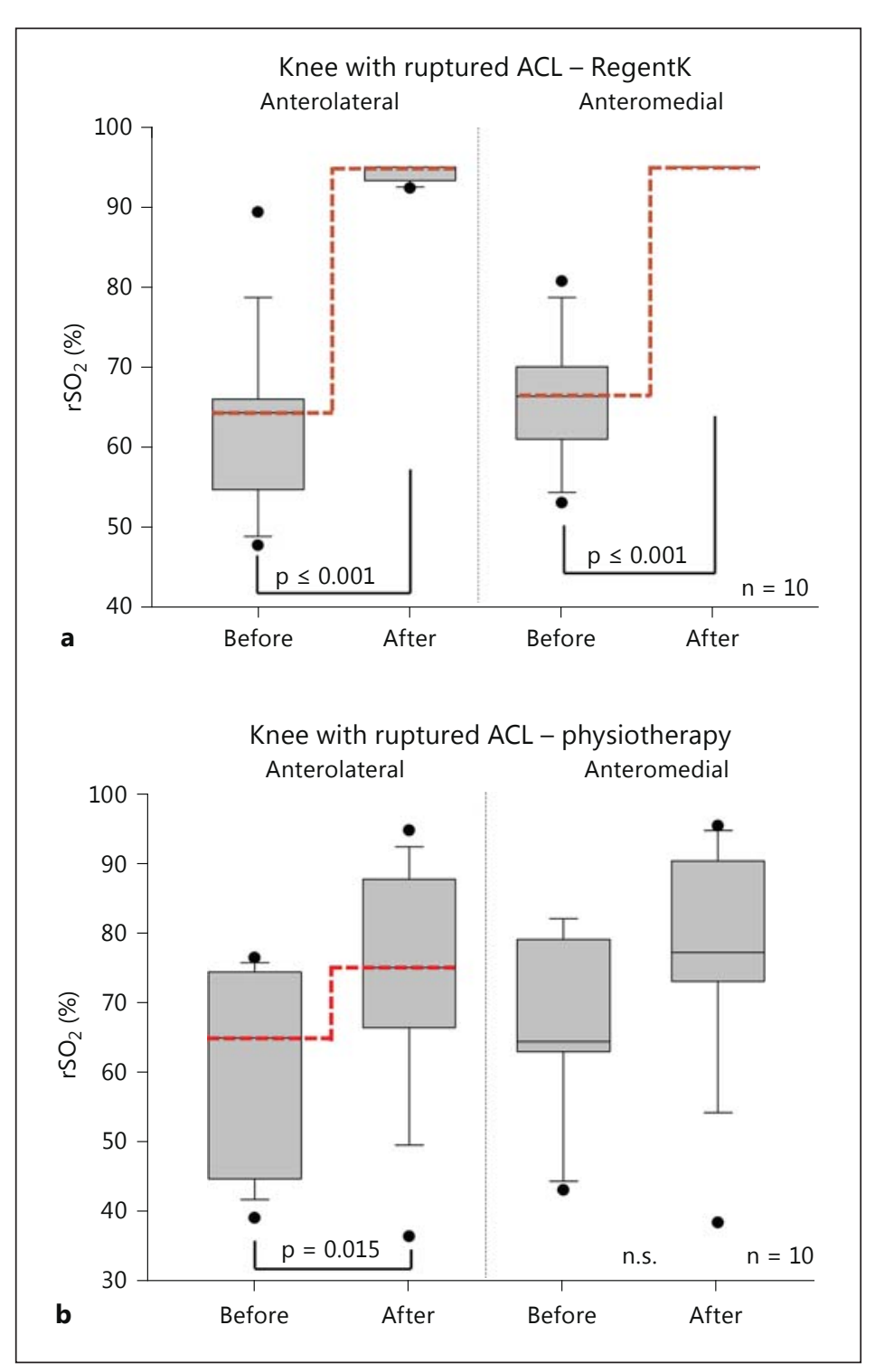

Fig. 4. Box plot presentation of changes in $\mathrm{rSO}_{2}$ in the injured knee after RegentK (a) and after physiotherapy (b) (ends of boxes: 25th and 75th percentile; horizontal line: median; error bars: 10 th and 90th percentile). Modified from Litscher et al. [5]. n.s. = Not significant.

ACL before and immediately after the manual therapy. Ten male patients were investigated with thermal imaging. An infrared camera operating at a wavelength range of 7.5-13 $\mu \mathrm{m}$ was used. Temperature was analysed at three locations on both knees and, in addition, on both feet. The study revealed that baseline temperature of the injured knee differed from that of the untreated control knee (fig. 5). After the therapy of the injured knee, the surface temperature was significantly increased in both knees (injured and control knee). There were no significant changes in the temperature of the feet [7].

In a second skin surface temperature study, the effects on the knee were investigated after RegentK compared to standard physiotherapy in patients with completely ruptured ACL. Twenty patients participated in this study. They were randomly assigned to group A (receiving RegentK) or group B (receiving physiotherapy). Each group again consisted of 10 patients. Temperature values were registered on four spots (three on the knee and one on the foot) of the injured and the healthy leg (control leg). The skin temperature increased significantly after RegentK on all sites of the injured leg, but after physiotherapy only the 
Litscher et al.: RegentK: The Scientific Investigation of a 40-Year-Old Method in Regenerative Medicine

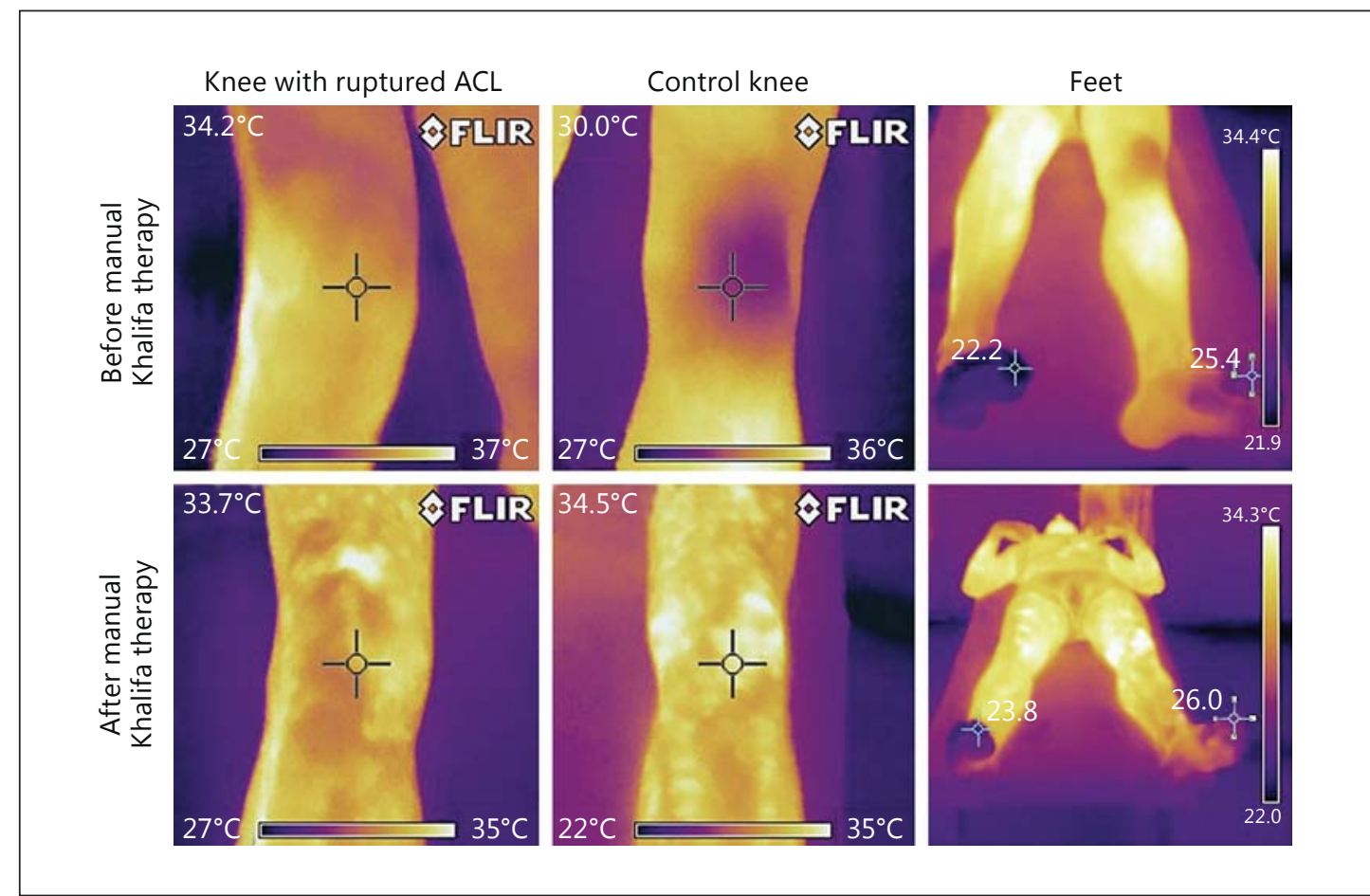

Fig. 5. Thermal images before (upper panel) and after (lower panel) RegentK (Khalifa therapy). Note the higher temperature in the injured knee compared to the control knee before treatment, whereas the temperature of the foot on the injured leg is initially lower than the temperature of the foot on the healthy leg (compare the colour of the toes). Modified from Litscher et al. [6].

measurement spots on the knee showed significant increases (fig. 6). After RegentK, the temperature had also increased significantly in the control leg, whereas in group B, the results were not significant [8].

\section{Electrodermal Mapping}

In a further study, a new system which measures and analyses electrical skin impedance in 48 channels within a $2.5 \times 3.5 \mathrm{~cm}$ matrix was used in rehabilitation medicine for the first time. This new technology has already been introduced and described [9]. Electrodermal activity was measured in 20 patients before and after the two different non-surgical treatments of a completely ruptured ACL. The first treatment was RegentK, the second a standard physiotherapy. The patients in the two groups were age matched, and all demographic data showed no significant differences. It was interesting that electrodermal activity was significantly decreased only after RegentK (fig. 7) [10].

Rupture of the ACL is a high-incidence injury usually treated surgically. According to common knowledge, it does not heal spontaneously, although some claim the opposite [11]. RegentK was developed for injuries of the musculoskeletal system by using specific pressure to the skin [11]. The therapy is described as functional-pathological. In this approach, function is the primary concern, not anatomy. The most important thing is not the ruptured ligament itself, but its function/dysfunction. RegentK restores the function of the knee in a natural way. During the 60-90 min of manual therapy, Mohamed Khalifa applies pressure to the injured 
Litscher et al.: RegentK: The Scientific Investigation of a 40-Year-Old Method in Regenerative Medicine

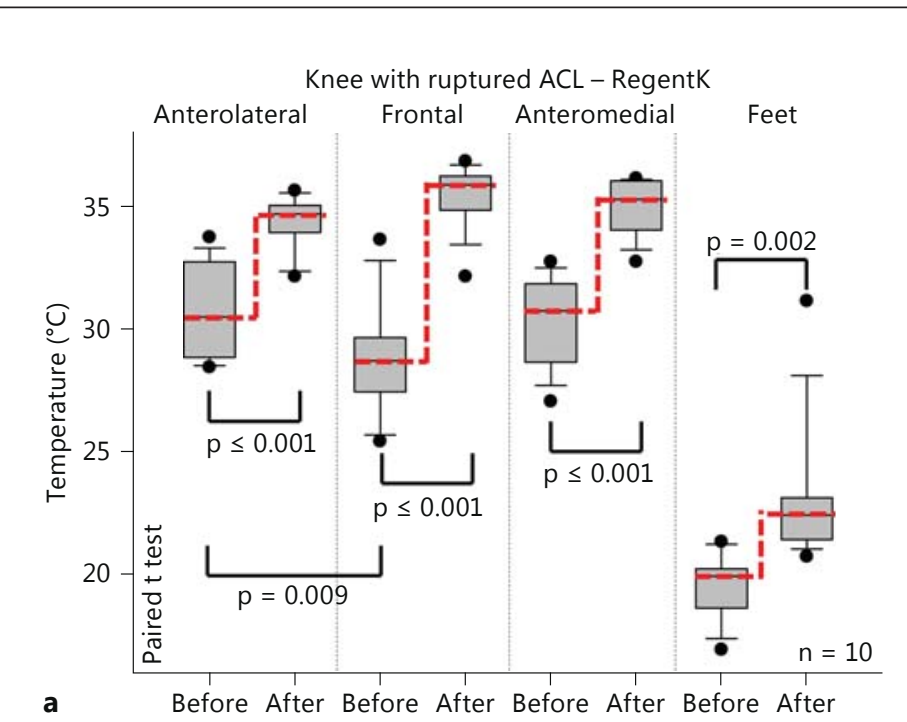

Fig. 6. Box plot presentation of changes in skin surface temperature on the injured knee after RegentK (a) and after physiotherapy (b). For further explanations, see figure 4. Modified from Litscher et al. [7]. n.s. = Not significant.

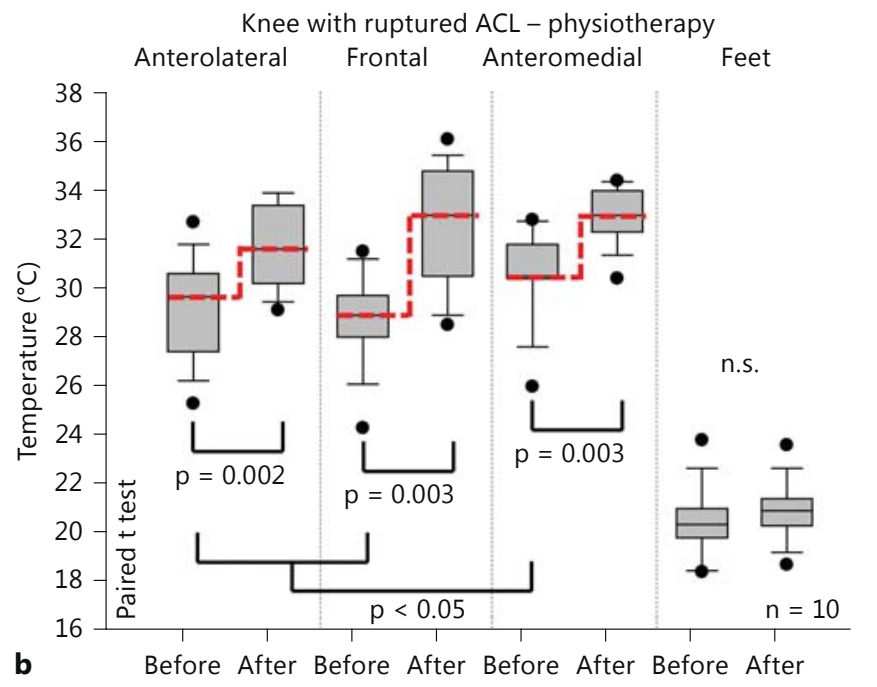

knee in order to activate the self-healing processes of the human body, using his hands as an instrument for both measurement and therapy. The force of the pressure is not comparable to that normally used in acupressure in Traditional Chinese Medicine [4]; it is much higher. In a randomised, controlled, observer-blinded, multicentre study, 30 patients with complete ACL rupture, verified by magnetic resonance imaging (MRI), were included [11]. Patients were randomised to receive either standardised physiotherapy or, additionally, $1 \mathrm{~h}$ of RegentK therapy at the first session. The initial status was comparable between both groups. Three months later, a control MRI and follow-up examinations were performed. There was a highly significant difference in the mean International Knee Documentation Committee (IKDC) score results. After 3 months, $47 \%$ of the RegentK patients, but no physiotherapy patient, demonstrated an end-to-end homogeneous ACL on MRI. We conclude that ACL healing can be improved with manual therapy. Physical activity can be performed without pain and a nearly normal range of motion after one treatment with specific pressure [11]. 
Litscher et al.: RegentK: The Scientific Investigation of a 40-Year-Old Method in Regenerative Medicine

Fig. 7. Graphical presentation of the changes in electrodermal activity on the lateral measurement position of the injured and control knee after RegentK (a) and physiotherapy (b). Modified from Litscher et al. [9]. n.s. = Not significant.

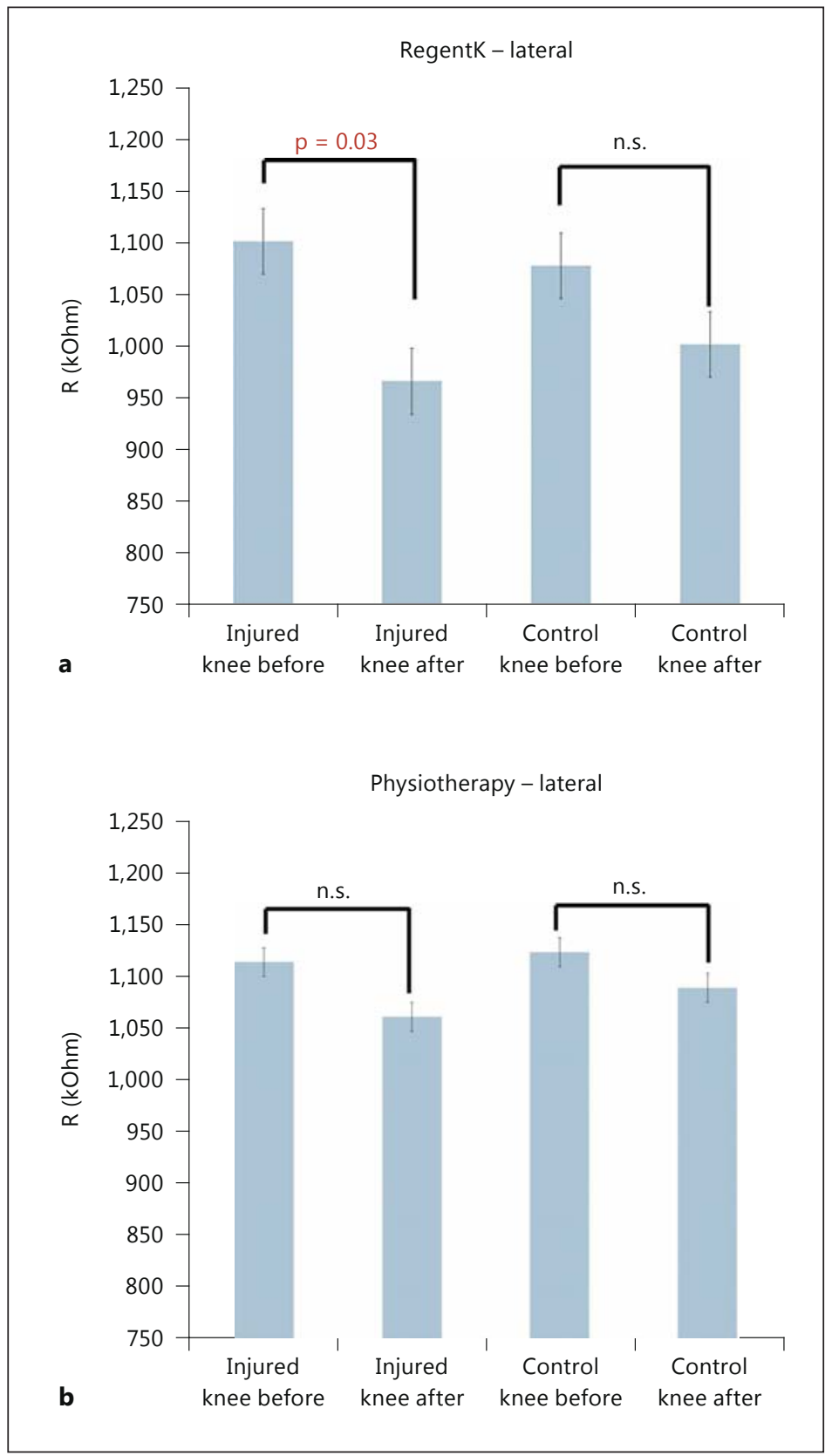

Pain- and injury-related massage, important in traditional Eastern medicine, is increasingly used in the Western world. Clearly, massage therapies are not totally devoid of risks. But the incidence of such events is low [12].

\section{Acknowledgements}

The study was supported by the Forschungsförderungsverein der Erkenntnisse von Mohamed Khalifa. The author would like to thank Ms Ingrid Gaischek, MSc, Medical University of Graz, for manuscript preparation. 
Litscher et al.: RegentK: The Scientific Investigation of a 40-Year-Old Method in Regenerative Medicine

\section{References}

1 RegentK - Regeneration Therapy after Khalifa. http://mohamed-khalifa.com (accessed June 22, 2015).

2 Tidy NM: Massage and Remedial Exercises in Medical and Surgical Conditions, ed 8. Bristol, John Wright and Sons Ltd, 1949, pp 77-78.

3 Niederführ G: Torn Ligaments? A Slipped Disc? Operations No Longer Necessary! Norderstedt, Books on Demand GmbH, 2002, pp 163-164.

4 Litscher G, Ofner M, He W, Wang L, Gaischek I: Acupressure at the meridian acupoint Xiyangguan (GB33) influences near-infrared spectroscopic parameters (regional oxygen saturation) in deeper tissue of the knee in healthy volunteers. Evid Based Complement Alternat Med 2013;2013:370341.

5 Litscher G, Ofner M, Litscher D: Manual Khalifa therapy in patients with completely ruptured anterior cruciate ligament in the knee: first results from near-infrared spectroscopy. N Am J Med Sci 2013;5:320-324.

6 Litscher D, Litscher G, Ofner M, Gaischek I, Malliga DE: Spectroscopic measurements in patients with completely ruptured anterior cruciate ligament before and after RegentK and physiotherapy. Integr Med Int 2014;1: 56-63.

7 Litscher G, Ofner M, Litscher D: Manual Khalifa therapy in patients with completely ruptured anterior cruciate ligament in the knee: first preliminary results from thermal imaging. N Am J Med Sci 2013;5:473-479.

-8 Litscher G, Litscher D, Ofner M, Gaischek I, Malliga DE: Thermal imaging in rehabilitation in patients with completely ruptured anterior cruciate ligament before and after RegentK and physiotherapy. Medicines 2014; $1: 12-21$.

-9 Litscher G, Wang L, Gao XY, Gaischek I: Electrodermal mapping: a new technology. World J Methodol 2011;1: 22-26.

10 Litscher G, Litscher D, Ofner M, Gaischek I, Malliga DE: RegentK and physiotherapy - electrodermal mapping. Medicines 2014;1:22-31.

11 Ofner M, Kastner A, Wallenböck E, Pehn R, Schneider F, Gröll R, Szolar D, Walach H, Litscher G, SandnerKiesling A: Manual Khalifa therapy improves functional and morphological outcome of patients with anterior cruciate ligament rupture in the knee: a randomized controlled trial. Evid Based Complement Alternat Med 2014;2014:462840.

12 Yin P, Gao N, Wu J, Litscher G, Xu S: Adverse events of massage therapy in pain-related conditions: a systematic review. Evid Based Complement Alternat Med 2014;2014:480956. 